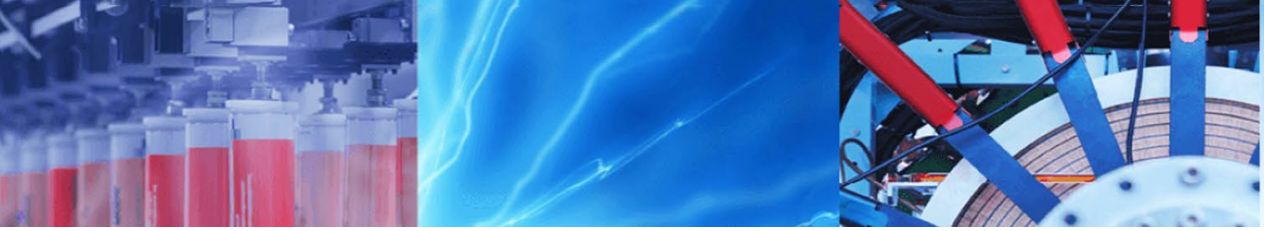

Research Article

\title{
A new approach for the optimal synthesis of four-bar path generator linkages
}

\author{
Neider Nadid Romero ${ }^{1} \cdot$ Alexandre Campos $^{2} \cdot$ Daniel Martins $^{3} \cdot$ Rodrigo S. Vieira ${ }^{3}$
}

Received: 30 July 2019 / Accepted: 17 October 2019 / Published online: 28 October 2019

(c) Springer Nature Switzerland AG 2019

\begin{abstract}
In this paper, an optimal synthesis of four-bar path generator, using a robust mathematical formulation is presented. Natural coordinates are used in order to solve the four-bar mechanism kinematic position analytically and the Hermitian conjugate is used to build a goal function whose range is the real numbers' set. A Teaching Learning Based Optimization Algorithm is implemented to test the proposed formulation robustness, also the possibility of extending the method to another type of mechanism is described. The main advantages of the formulation are its simplicity and robustness due that the equations involved in the formulation are algebraic and the numerical field is the complex's set.
\end{abstract}

Keywords Optimal synthesis · Four-bar mechanism · Natural coordinates · Hermitian conjugate

\section{Introduction}

Several Four-bar linkage applications are found in Robotics. Han et al. [8], suggest the six degree-of-freedom robot leg using the four-bar linkage mechanism with high rigidity to minimize the actuators weight in a bipedal walking robot. Additionally, robot end-effector, that actually interacts with the object, design is of high importance. Some end-effector are grippers based on Four-bar linkages [20]. Some surgical and field robots based on Fourbar linkage, that no require actuators which are attached directly to driving joints and that may be independently controlled, are presented in Hoyul and Youngjin [9].Displacement analysis for Four-bar linkages, has been extensively reported in the technical literature $[6,7,12,15,21]$.

With regard to optimization bio-inspired techniques have been increased considerably in the last two decades. One of the earliest works in evolutionary algorithm applied to the optimal synthesis of four-bar path generator is reported by Cabrera et al. [3] . The authors developed a genetic algorithmic to solve three study cases with and without prescribed timing and considering different target points. In Nariman-Zadeh et al. [22] a path synthesis procedure to generator linkages using a neural network is proposed, it consists of a learning stage where a large number of kinematic simulation are generated with random dimension, and in the second stage the neural network is applied to approximate a synthesis problem solution. Bulatović and Dordević [1] describe the process of optimal synthesis of a four-bar linkage using the controlled deviations method of the variables with the differential evolution algorithm application. In [16] authors deals the Pareto optimal synthesis of four-bar mechanisms for path generation considering tracking error and transmission angle error, it is solved using a multi-objective hybrid genetic algorithm. A hybrid evolutionary algorithm for path synthesis of four-bar linkage is presented in [13], here the hybridization between a genetic algorithm and a differential evolution algorithm is proposed. The authors state that the main advantages of this algorithm are the

$\triangle$ Neider Nadid Romero, neider.nadid@gmail.com; Alexandre Campos, alexandre.campos@udesc.br; Daniel Martins, danielemc@ gmail.com; Rodrigo S. Vieira, rodrigo.vieira@ufsc.br | ${ }^{1}$ Mechanical Engineering Department, Federal University of Santa Catarina, Centro Tecnologico, Florianópolis, SC, Brazil. ${ }^{2}$ UDESC, Florianópolis, SC, Brazil. ${ }^{3}$ University of Santa Catarina, Centro Tecnologico, Florianópolis, SC, Brazil. 
simplicity and ease to implement and solve complicated real-world optimization problems, with no need of deep knowledge of the search space. In [10] authors present a novel approach to the multi-objective optimal path synthesis of four-bar linkages and applying it to the traditional problem with one, two and three objective function . A novel algorithm called Malaga University Mechanism Synthesis Algorithm for path synthesis of mechanisms is successfully applied to six cases of path and function synthesis of four-bar and six-bar mechanisms [4]. Related subjects, see the title of the papers $[2,5,11]$. In the literature, the kinematic and optimization formulation of the four-bar path generator is very similar. The kinematic formulation in these works are based on the traditional closed-loop condition and the goal function is the sum of the square of the Euclidean distances, where the main difficulty is the penalization needing when the kinematic does not have solution in the two-dimensional real space. For this reason, the formulation here proposed is based in the use of the natural coordinates and the Hermitian conjugate operator in order to build an objective function whose output is always a positive real number. It should also be noted that the formulation proposed here can be extended to any problem of planar mechanisms synthesis with closed solution. The paper is organized as follows: Chapter 2 deals about the analytical position solving using natural coordinates. In chapter 3 the optimization problem is formulated using the Hermitian conjugate operator. Chapter 4 shows the mathematical description of teaching learning based optimization algorithm. In chapter 5 a result for three path synthesis problem is shown and compared with the literature. Finally, chapter 6 content the paper conclusions.

\section{Position analysis of the four-bar mechanism}

The Fig. 1 shows a schematic four-bar mechanism, where $P$ is the coupler point and $\varphi$ is the input angle. Here the position problem is solved using natural coordinates $[17,19]$.

The vector of natural coordinates is

$$
q=\left[\begin{array}{l}
C \\
D \\
P
\end{array}\right]=\left[\begin{array}{l}
x_{C} \\
y_{C} \\
x_{D} \\
y_{D} \\
x_{P} \\
y_{P}
\end{array}\right]
$$

where the point $C$ can be computed as,

$C=\left[\begin{array}{l}x_{A}+a \cos \varphi \\ y_{A}+a \sin \varphi\end{array}\right]$

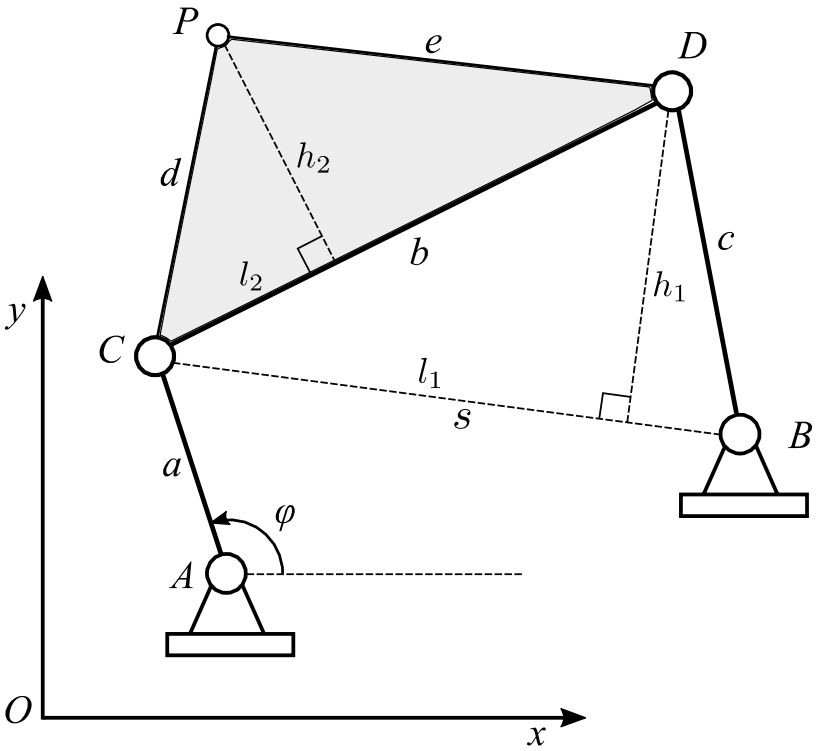

Fig. 1 Kinematic modelling of the four-bar mechanism through natural coordinates

and from mechanism's geometry it is derived the following relationships:

$s=\|B-C\| \quad l_{1}=\frac{b^{2}+s^{2}-c^{2}}{2 s} \quad h_{1}=t_{1} \sqrt{b^{2}-l_{1}^{2}}$

where $t_{1} \in\{-1,1\}$. The geometric variable can be written in compact form as

$N_{1}=\left[\begin{array}{ll}\frac{l_{1}}{s} & -\frac{h_{1}}{s} \\ \frac{h_{1}}{s} & \frac{l_{1}}{s}\end{array}\right]$

therefore the point $D$ is determined by

$D=C+N_{1}(B-C)$

Moreover, the geometric variables in the coupler are related by the equations

$I_{2}=\frac{d^{2}+b^{2}-e^{2}}{2 b} \quad h_{2}=t_{2} \sqrt{d^{2}-I_{2}^{2}}$

where $t_{2} \in\{-1,1\}$. The geometric variables are grouped in the matrix

$N_{2}=\left[\begin{array}{ll}\frac{l_{2}}{b} & -\frac{h_{2}}{b} \\ \frac{h_{2}}{b} & \frac{l_{2}}{b}\end{array}\right]$

thereby the point $P$ is computed as

$P=C+N_{2}(D-C)$

The values of variables $t_{1}$ and $t_{2}$ determine the configurations or the assemble modes of the mechanism. The Fig. 2 


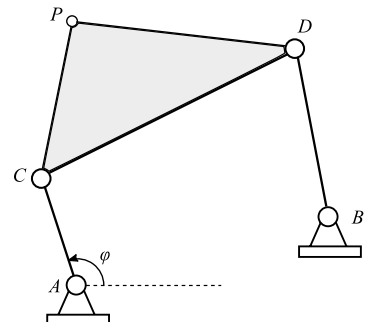

(a)

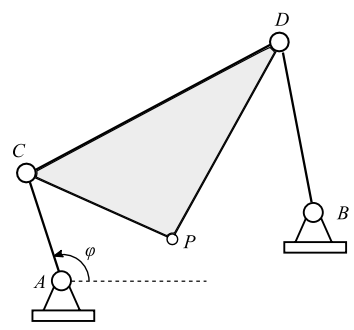

(c)

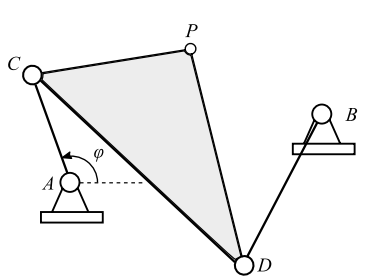

(b)

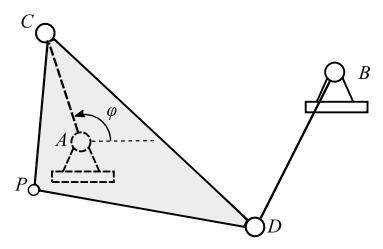

(d)
Fig. 2 Possible configuration of a four-bar mechanism

shows four possible configuration for the mechanism, where $t_{1}=1$ and $t_{2}=1$ represent the configuration showed in the Fig. $2 \mathrm{a}, t_{1}=-1$ and $t_{2}=1$ represent the configuration showed in the Fig. $2 \mathrm{~b}, t_{1}=1 \mathrm{and} t_{2}=-1$ represent the configuration showed in the Fig. $2 \mathrm{c}$ and $t_{1}=-1, t_{2}=-1$ represent the configuration showed in the Fig. $2 \mathrm{~d}$.

In general the points $D$ and $P$ belong to the two-dimensional complex space, thus the mechanism's assembles only is possible if all the components of and are real numbers. The principle approach in this paper is to work in field of the complex numbers in order to make the optimization method robust.

\section{Optimization problem formulation}

\subsection{The Hermitian conjugate}

If $Z$ is an $m \times n$ matrix with complex entries, then the Hermitian conjugate of $Z$, denoted by $Z^{*}$, is defined by

$Z^{*}=(\bar{Z})^{T}$

that is, if $Z=\left(z_{i j}\right)$, then $\bar{Z}=\left(\overline{z_{i j}}\right)$ and $Z^{*}=(\bar{Z})^{T}$ [14]. In this work the Hermitian conjugate is used to define the synthesis error in the following way

$\varepsilon=\left({ }^{d} P-P\right)^{*}\left({ }^{d} P-P\right)$

where ${ }^{d} P$ is the desired point and $P$ is the coupler point reached by the mechanism.

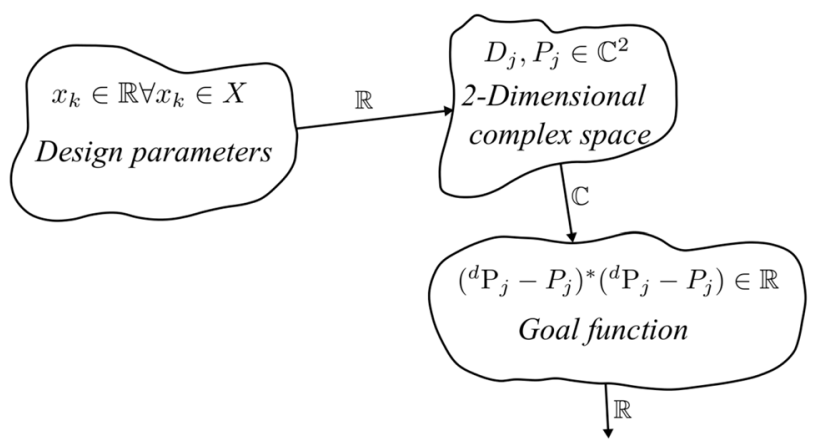

Fig. 3 Illustration of computing of the goal function

\subsection{Goal function and constraints}

The goal function is defined as the sum of the synthesis error determined by Eq. (6). In the Fig. 3 the computing of the goal function is illustrated. The design parameters are real values, thus with this parameter the position problem is solved, where the natural coordinates belong to the twodimensional complex space and finally the goal function is computed yielding a real value.

The optimization problem is formulated as

$\min f(X)=\sum_{j=1}^{n}\left({ }^{d} P_{j}-P_{j}\right) *\left({ }^{d} P_{j}-P_{j}\right)$

s.t: a) $2[\min (a, b, c, f)+\max (a, b, c, f)]-(a+b+c+f)<0$

b) $\varphi_{j}-\varphi_{j+1}<0$

c) $\min (a, b, c, f)-a=0$

d) $x_{k} \in\left[L i_{k}, L s_{k}\right] \forall x_{k} \in X$

where $f=\|B-A\|$ and the design variables are

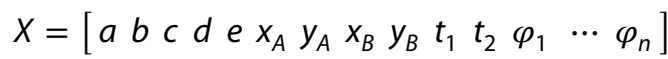

In the Eq. (7) the constraint a) guarantees the Grashof condition, the constraint b) guarantees a crescent angle sequence, c) warrants that input link be the shortest and d) is the lower and upper bound of the design variables. For solving the optimization problem using an evolutionary technique in this work a penalty function is used, thus the optimization problem is reformulated as

$\min f(X)=\sum_{j=1}^{n}\left({ }^{d} P_{j}-P_{j}\right) *\left({ }^{d} P_{j}-P_{j}\right)+P(X)$

$P(X)=\rho_{1} \cdot g_{1}(X)+\rho_{2} \cdot g_{2}(X)+\rho_{3} \cdot g_{3}(X)$ 
$g_{1}(X)=\left\{\begin{array}{l}1 \text { if } \varphi_{j}-\varphi_{j+1}=0 \\ {\left[\max \left(0,\left(\varphi_{j}-\varphi_{j+1}\right)\right)\right]^{2}}\end{array}\right.$

$g_{2}(X)=\left[\max \left(0, r_{2}(X)\right)\right]^{2}$

$g_{3}(X)=[\min (a, b, c, f)-a]^{2}$

where $P(X)$ is a penalty function, $\rho_{1}, \rho_{2}, \rho_{3}$ are constants of a very high value that penalize the goal function when the associated constraint fails and

$r_{2}(X)=2[\min (a, b, c, f)+\max (a, b, c, f)]-(a+b+c+f)$

\section{Optimization implementation}

\subsection{Teaching learning based optimization algorithm}

A teaching learning based optimization (TLBO) is a teaching-learning process inspired algorithm proposed by Rao et al. [18]. This algorithm consists of two phases, Teacher phase and Learner phase.

In the teaching phase a teacher tries to increase the mean result of the class in the subject taught by him depending on his capability, it is written mathematically as

$X_{j, k, i}^{\prime}=X_{j, k, i}+$ Difference_Mean ${ }_{j, k, i}$

where $X_{j, k, i}$ is the result of the $k$ learner in subject or design variable $j$ in the iteration $i, X_{j, k, i}^{\prime}$ is the updating of $X_{j, k, i}$ and

Difference_Mean ${ }_{j, k, i}=r_{i}\left(X_{j . k b e s t, i}-T_{F} M_{j, i}\right)$

where $r_{i}$ is a random number in the range $[0,1], X_{j, k b e s t, i}$ is the result of the best learner in subject $j, T_{F}$ is a value that can be either 1 or 2 and computed as

$T_{F}=\lfloor 1+\operatorname{rand}(0,1)\{2-1\}\rceil$

finally $M_{j, i}$ is the mean result of the learners in subject $j$.

In the learning phase the interaction among learners increases their knowledge. The interchanging of knowledge between two learners is computed as

$X_{j, P, i}^{\prime \prime}=X_{j, P, i}^{\prime}+r_{i}\left(X_{j, P, i}^{\prime}-X_{j, Q, i}^{\prime}\right)$ if $X_{\text {total }-P, i}^{\prime}<X_{\text {total }-Q, i}^{\prime}$

$X_{j, P, i}^{\prime \prime}=X_{j, P, i}^{\prime}+r_{i}\left(X_{j, Q, i}^{\prime}-X_{j, P, i}^{\prime}\right)$ if $X_{\text {total }-Q, i}^{\prime}<X_{\text {total }-P, i}^{\prime}$

where $P$ and $Q$ are randomly selected, $X_{\text {total }-P, i}^{\prime}$ and $X_{\text {total }-Q, i}^{\prime}$ are the updated function values of $X_{\text {total }-P, i}$ and $X_{\text {total }-Q, i}$ respectively, at the end of teacher phase.
The Fig. 4 shows the flowchart of TLBO that is a population-based algorithm which simulates the teaching-learning process of the class room. his algorithm requires only the common control parameters such as the population size and the number of generations and does not require any algorithm-specific control parameters [18].

\subsection{Treatment of design variables}

The TLBO here descripted works with real variables, but the vector of design variables Eq. (12) contain integer variables that determine the configuration of the mechanism. Therefore, to deal with integer design variables the follow auxiliary vector design is used

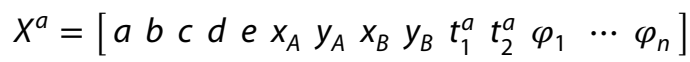

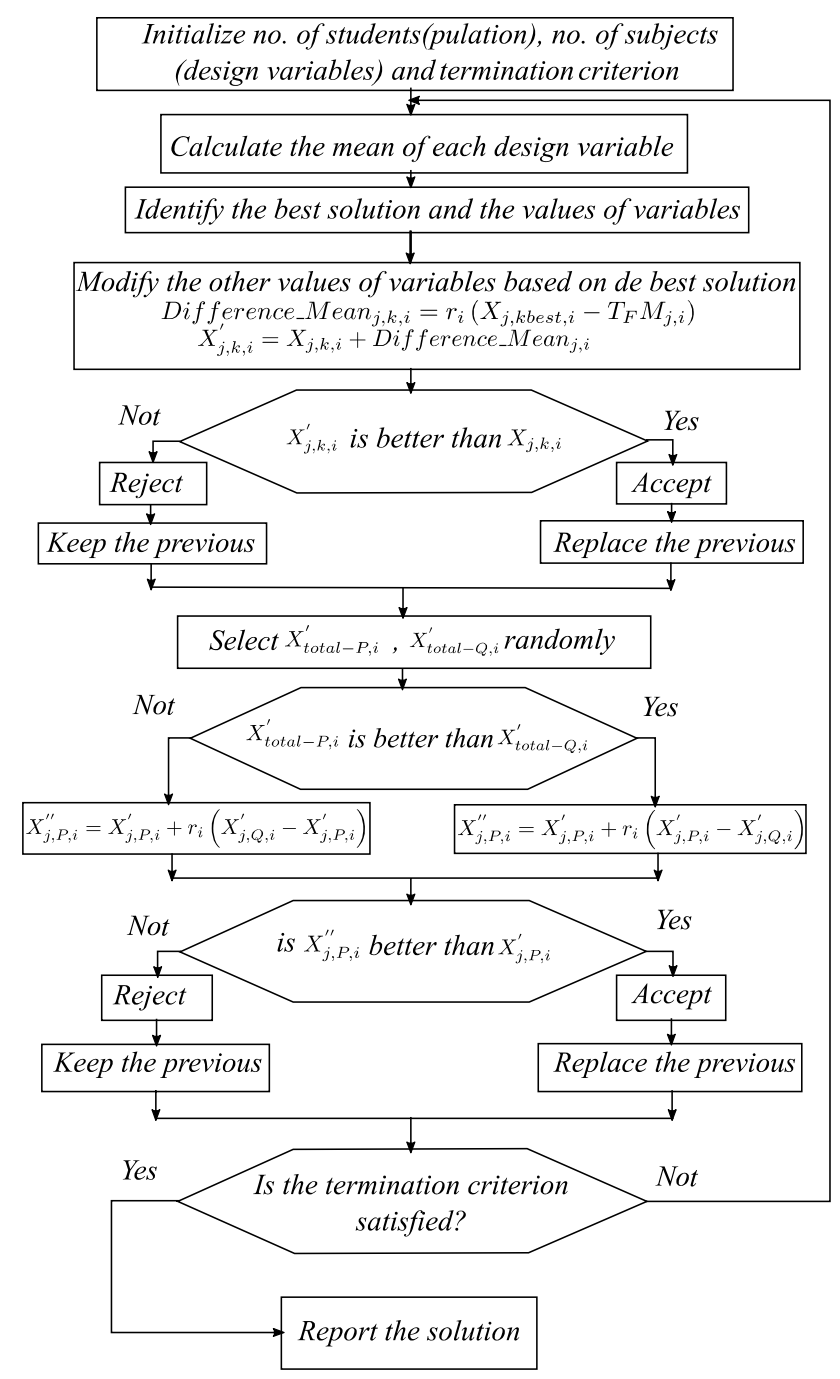

Fig. 4 Flowchart of TLBO algorithm. Adapted from [18] 
where $t_{1}^{a}$ and $t_{2}^{a}$ are the auxiliary variables of the $t_{1} t_{2}$ respectively and limited to the interval $[0,1]$. The auxiliary variables are transformed using the equation

$t_{i}=(-1)^{\left\lfloor t_{i}^{a}\right\rfloor} \quad i \in\{1,2\}$

\section{Results}

In this section three problem proposed in the literature are solved using the procedure developed in the previous sections. A processor Intel i5-4300U CPU @ $2.5 \mathrm{GHz}$ was used to program the solutions implemented in $\mathrm{Matlab}^{\circledR}$ and the solutions found are showed and compared with the literature.

\subsection{Problem 1}

This problem was taken from [3] which considers six-point path synthesis aligned without prescribe timing.

Design variables are:

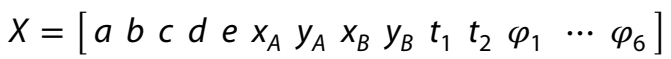

Target points are:

${ }^{d} P_{j}=[(20,20),(20,25),(20,30),(20,35),(20,40),(20,45)]$

Limits of variables:

$a, b, c, d, e \in[0,60]$

$x_{A}, y_{A}, x_{B}, y_{B} \in[-60,60]$

$t_{1}, t_{2} \in\{-1,1\}$

$\varphi_{1}, \varphi_{2}, \varphi_{3}, \varphi_{4}, \varphi_{5}, \varphi_{6} \in\left[0,360^{\circ}\right]$

The Fig. 5 shows the mechanism obtained with the approach here proposed as well as the mechanism found by Cabrera et al. [3].
The values of design variables are:

$$
\begin{aligned}
& a=8.9169 b=32.6786 c=60 d=45.4871 e=16.2315 \\
& x_{A}=53.9887 y_{A}=4.9096 x_{B}=-29.5025 y_{B}=2.9867 \\
& t_{1}=-1 t_{2}=-1\left\{\varphi_{i}\right\} \\
& =\{0.0006,22.0215,37.3841,53.3718,71.6155,95.6007\}
\end{aligned}
$$

\subsection{Problem 2}

This problem is about twelve-point path synthesis without prescribed timing, it was taken from [2].

Design variables are:

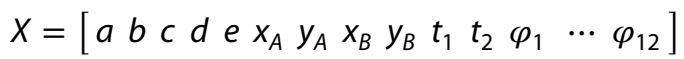

Target points are:

${ }^{d} P_{j}=\left[\begin{array}{l}(4.15,2.21),(4.5,2.18),(4.53,1.83),(4.13,1.68) \\ (3.67,1.58),(2.96,1.33),(2.67,1.06),(2.63,0.82) \\ (2.92,0.81),(3.23,1.07),(3.49,1.45),(3.76,1.87)\end{array}\right]$

Limits of variables:

$a, b, c, d, e \in[0,50]$

$x_{A}, y_{A}, x_{B}, y_{B} \in[-20,20]$

$t_{1}, t_{2} \in\{-1,1\}$

$\varphi_{1}, \varphi_{2}, \varphi_{3}, \varphi_{4}, \varphi_{5}, \varphi_{6}, \varphi_{7}, \varphi_{8}, \varphi_{9}, \varphi_{10}, \varphi_{11}, \varphi_{12} \in\left[0,360^{\circ}\right]$

The optimized mechanism found by Bulatović et al. [10] and the optimized mechanism obtained with the approach here proposed is showed in the Fig. 6 .

The values of design variables are:
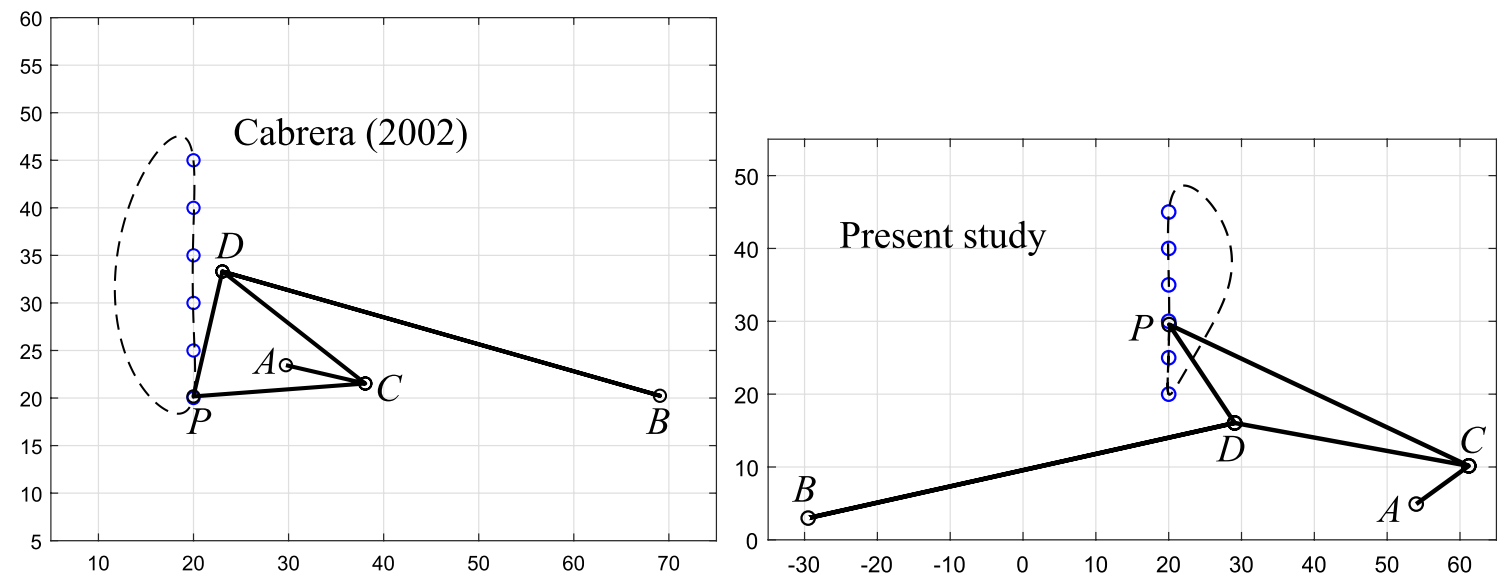

Fig. 5 Solution to the problem 1 

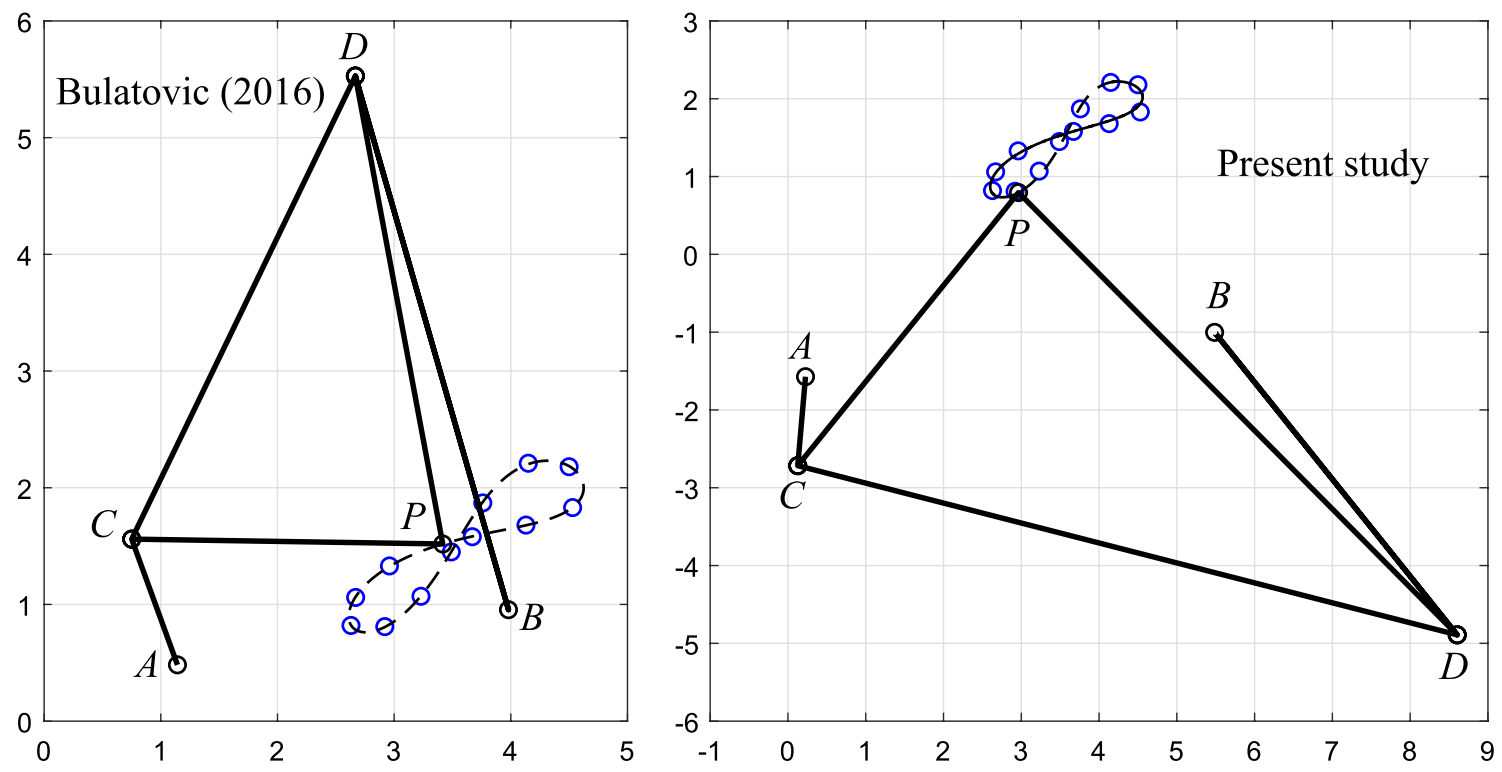

Fig. 6 Solution to the problem 2

$a=1.1481 b=8.753 c=4.9843 d=4.5143 e=8.008$

Target points are:

$x_{A}=0.2256 y_{A}=-1.5728 x_{B}=5.4858 y_{B}=-1.003 t_{1}=-1$

$t_{2}=1$

$\left\{\varphi_{i}\right\}=\left\{\begin{array}{c}13.0309,35.9183,66.1241,94.3757,117.3105,157.358 \\ 185.143,217.588,263.420,291.349316 .623,341.74\end{array}\right\}$

${ }^{d} P_{j}=\left[\begin{array}{l}(20,10),(17.66,15.142),(11.736,17.878),(5,16.928) \\ (0.60307,12.736),(0.60307,7.2638),(5,3.0718) \\ (11.736,2.1215),(17.66,4.8577),(20,10)\end{array}\right]$

Limits of variables:

\subsection{Problem 3}

$a, b, c, d, e \in[0,80]$

$x_{A}, y_{A}, x_{B}, y_{B} \in[-80,80]$

$t_{1}, t_{2} \in\{-1,1\}$

The Fig. 7 shows the mechanism obtained with the $\varphi_{1}, \varphi_{2}, \varphi_{3}, \varphi_{4}, \varphi_{5}, \varphi_{6}, \varphi_{7}, \varphi_{8}, \varphi_{9}, \varphi_{10} \in\left[0,360^{\circ}\right]$ by Cabrera et al. [4]. Design variables are:

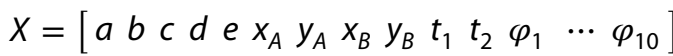
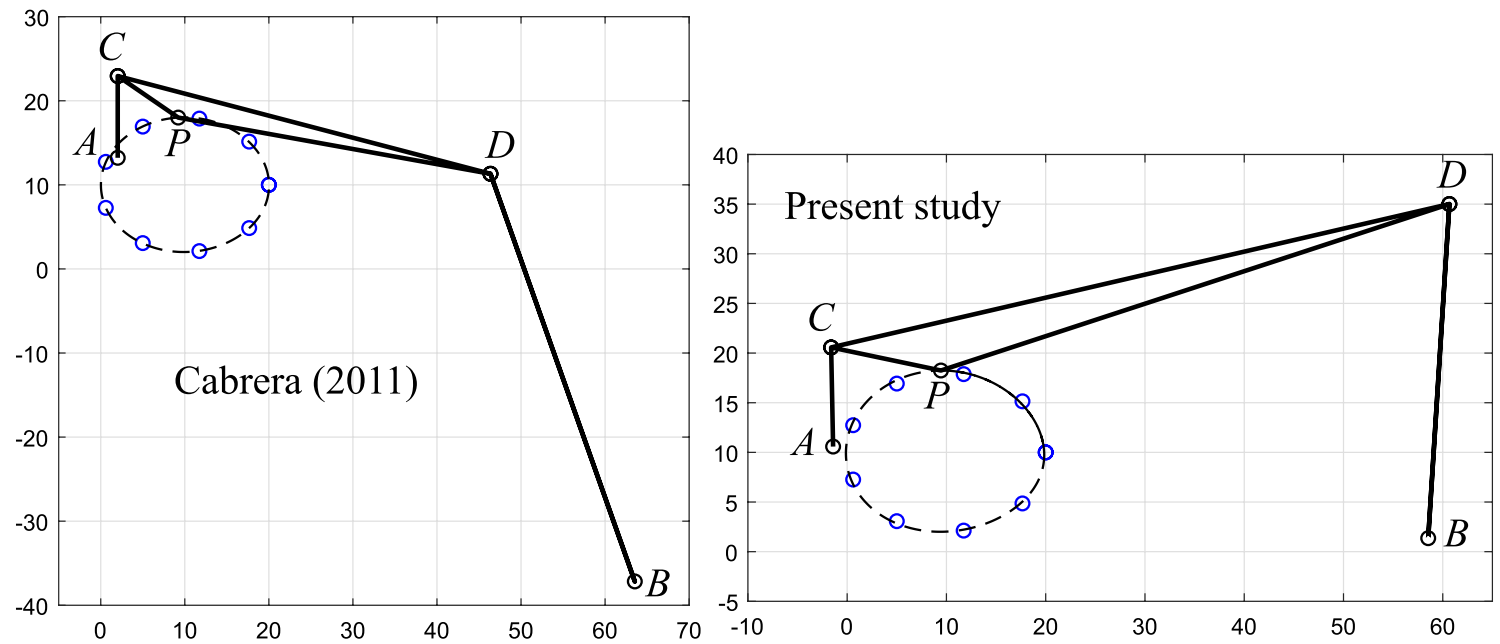

Fig. 7 Solution to the problem 3 
The values of design variables are:

$a=9.9864 b=63.93 c=33.7178 d=11.304 e=53.8921$

$x_{A}=-1.4159 y_{A}=10.5839 x_{B}=58.532 y_{B}=1.358 t_{1}=1$

$t_{2}=-1$

$\left\{\varphi_{i}\right\}=\left\{\begin{array}{c}1.1317,38.653777 .7545,118.224,158.8833 \\ 198.7785,239.8071,281.6572,320.3808,360\end{array}\right\}$

\subsection{Discussion of the method}

The proposed procedure is focused on the formulation of the optimization problem and not on the optimization method. Where the main difference of the proposed procedure with the methods known to the authors is that the synthesis error is always a real number independent of the values of the design variables, this allows that individuals potentially close to the global optimum are not eliminated. The results of the optimization of the three problems are comparable with those of the literature and moreover with different dimensions and configurations.

It should also be noted that the proposed procedure encompasses a greater number of potential solutions unlike other procedures in the literature. This is due to the fact that the objective function is never penalized with values too large when the mechanism assembly is impossible even if the dimensions are close to their optimal values.

\section{Conclusion}

This paper presents a new approach for the optimal path synthesis of a four-bar linkage. Where the robustness of the procedure is based on the analytical solution of the mechanism's position through the use of natural coordinates and their operation under the field of the set of complex numbers. Further to the construction of the objective function using the Hermitian conjugate operator that allows defining define a synthesis error that is always a real value.

As an optimization method, a Teaching Learning Based Optimization Algorithm was modified due that originally the algorithm only worked with continuous variables. The modified TLBO was implemented in Matlab ${ }^{\circledast}$ for three trajectory synthesis problems with six, twelve and ten precision points respectively.

The proposed procedure can be used for the synthesis of any planar mechanism whose position can be obtained analytically, and using any method of evolutionary optimization.
Acknowledgements This work was partially supported by Fundação Coordenação de Aperfeiçoamento de Pessoal de Nível Superior (CAPES) PGPTA 59/2014 AUXPE 3686/2014.

\section{Compliance with ethical standards}

Conflict of interest The authors declare that they have no competing interest. Authors take responsibility for all the content of this work.

\section{References}

1. Bulatović RR, Dordević SR (2009) On the optimum synthesis of a four-bar linkage using differential evolution and method of variable controlled deviations. Mech Mach Theory 44(1):235-246. https://doi.org/10.1016/j.mechmachtheory.2008.02.001

2. Bulatović RR, Miodragović G, Bošković MS (2016) Modified Krill Herd (MKH) algorithm and its application in dimensional synthesis of a four-bar linkage. Mech Mach Theory 95:1-21. https ://doi.org/10.1016/j.mechmachtheory.2015.08.004

3. Cabrera J, Simon A, Prado M (2002) Optimal synthesis of mechanisms with genetic algorithms. Mech Mach Theory 37(10):11651177. https://doi.org/10.1016/S0094-114X(02)00051-4

4. Cabrera JA, Ortiz A, Nadal F, Castillo JJ (2011) An evolutionary algorithm for path synthesis of mechanisms. Mech Mach Theory 46(2):127-141. https://doi.org/10.1016/j.mechmachth eory.2010.10.003

5. Chanekar PV, Fenelon MAA, Ghosal A (2013) Synthesis of adjustable spherical four-link mechanisms for approximate multipath generation. Mech Mach Theory 70:538-552. https://doi. org/10.1016/j.mechmachtheory.2013.08.009

6. Crane C, Duffy J (1998) Kinematic analysis of robot manipulators. Cambridge University Press, Cambridge

7. Dukkipati RV (2001) Spatial mechanisms: analysis and synthesis. CRC Press, Boca Raton

8. Han S, Um S, Kim S (2016) Mechanical design of robot lower body based on four-bar linkage structure for energy efficient bipedal walking. In: 2016 IEEE international symposium on safety, security, and rescue robotics (SSRR). IEEE, pp 402-407. https://doi.org/10.1109/SSRR.2016.7784334

9. Hoyul L, Youngjin C (2010) Stackable 4-BAR mechanisms and their robotic applications. In: 2010 IEEE/RSJ international conference on intelligent robots and systems. IEEE, pp 2792-2797. https://doi.org/10.1109/IROS.2010.5651921

10. Khorshidi M, Soheilypour M, Peyro M, Atai A, Shariat Panahi M (2011) Optimal design of four-bar mechanisms using a hybrid multi-objective GA with adaptive local search. Mech Mach Theory 46(10):1453-1465. https://doi.org/10.1016/j.mechmachth eory.2011.05.006

11. Kim BS, Yoo HH (2014) Body guidance syntheses of four-bar linkage systems employing a spring-connected block model. Mech Mach Theory 85:147-160. https://doi.org/10.1016/j.mechm achtheory.2014.11.022

12. Kreutzinger R (1942) über die bewegung des schwerpunktes beim kurbelgetriebe. Getriebetechnik 10(9):397-398

13. Lin WY (2010) A GA-DE hybrid evolutionary algorithm for path synthesis of four-bar linkage. Mech Mach Theory 45(8):10961107. https://doi.org/10.1016/j.mechmachtheory.2010.03.011

14. Martin A, Harvey M (2012) Linear algebra, concepts and methods. Cambridge University Press, The London School of Economics and Political Science, Cambridge

15. McCarthy JM, Soh GS (2010) Geometric design of linkages, vol 11. Springer, Berlin 
16. Nariman-Zadeh N, Felezi M, Jamali A, Ganji M (2009) Pareto optimal synthesis of four-bar mechanisms for path generation. Mech Mach Theory 44(1):180-191. https://doi.org/10.1016/j. mechmachtheory.2008.02.006

17. Neider R (2016) Análisis de posición de un mecanismo de cuatro barras utilizando coordenadas naturales. Rev Iberoam Ing Mec 20:83-90

18. Rao RV (2016) Teaching learning based optimization algorithm. Springer, Cham. https://doi.org/10.1007/978-3-319-22732-0

19. Romero N, Flórez E, Mendoza L (2017) Optimization of a multilink steering mechanism using a continuous genetic algorithm. J Mech Sci Technol. https://doi.org/10.1007/s12206-017-0607-1
20. Saha DT, Sanfui S, Kabiraj R, Das DS (2014) Design and implementation of a 4-bar linkage gripper. IOSR J Mech Civ Eng 11(5):61-66. https://doi.org/10.9790/1684-11546166

21. Suh $\mathrm{CH}$, Radcliffe CW (1978) Kinematics and mechanisms design. Wiley, New York

22. Vasiliu A, Yannou B (2001) Dimensional synthesis of planar mechanisms using neural networks: application to path generator linkages. Mech Mach Theory 36:299-310. https://doi. org/10.1016/S0094-114X(00)00037-9

Publisher's Note Springer Nature remains neutral with regard to jurisdictional claims in published maps and institutional affiliations. 